\title{
BOUNDARY LAYER ANALYSIS AND QUASI-NEUTRAL LIMITS IN THE DRIFT-DIFFUSION EQUATIONS*
}

\author{
Yue-Jun PenG ${ }^{1}$
}

\begin{abstract}
We deal with boundary layers and quasi-neutral limits in the drift-diffusion equations. We first show that this limit is unique and determined by a system of two decoupled equations with given initial and boundary conditions. Then we establish the boundary layer equations and prove the existence and uniqueness of solutions with exponential decay. This yields a globally strong convergence (with respect to the domain) of the sequence of solutions and an optimal convergence rate $O\left(\varepsilon^{\frac{1}{2}}\right)$ to the quasi-neutral limit in $L^{2}$.

Résumé. On étudie les couches limites et les limites de quasi-neutralité aux systèmes de dérivéediffusion. On montre d'abord que cette limite est unique et déterminée par un système découplé avec données initiales et aux limites. On établit ensuite les équations des couches limites et montre l'existence et l'unicité de solutions avec l'atténuation exponentielle. Ceci implique un résultat de convergence globale (par rapport au domaine) de la suite de solutions et un taux de convergence optimale $O\left(\varepsilon^{\frac{1}{2}}\right)$ dans la limite de quasi-neutralité dans $L^{2}$.
\end{abstract}

Mathematics Subject Classification. 35B25, 35B40, 35K57.

Received: May 9, 2000. Revised: December 6, 2000.

\section{INTRODUCTION}

The drift-diffusion equations are fundamental models for the mathematical description and numerical simulation of plasmas physics and semiconductor devices. These equations consist of the continuity equations for particle densities and a Poisson equation for electrostatic potential. Since they are elliptic-parabolic type, we consider them in an open and bounded domain with initial conditions and mixed Dirichlet-Neumann boundary conditions. The existence of solutions to these equations has been proved under natural assumptions. In some situation, the uniqueness of solutions is also obtained, see $[7,10]$. On the other hand, in numerical simulations the Euler-Poisson equations are often replaced by the drift-diffusion equations to simplify computations. This approximation is valid as the relaxation time is small. The mathematical justification of this zero-relaxation-time limit has been rigorously performed in $[11,13]$.

In $[12,14]$, we are interested in some asymptotic limits in the drift-diffusion equations. More precisely, the zero-electron-mass limit and the quasi-neutral limit are studied. The first limit is proved under a compatibility condition on the Dirichlet boundary data which excludes the boundary layer phenomena. However, the second

Keywords and phrases. Asymptotic analysis, boundary layers, optimal convergence rate, drift-diffusion equations.

* This research was partially supported by Zheng Ge Ru Foundation.

1 Laboratoire de Mathématiques Appliquées, CNRS UMR 6620, Université Blaise Pascal (Clermont-Ferrand 2), 63177 Aubière Cedex, France, and, The Institute of Mathematical Sciences, The Chinese University of Hong Kong, Shatin NT, Hong Kong. 
limit is obtained even in the presence of the boundary layers. Both proofs rely on the use of the so-called entropy functional which yields appropriate uniform estimates to apply compensated compactness arguments. In this paper, we give an analysis of these boundary layers in the quasi-neutral limit.

We consider an ensemble of particles consisting of electrons with density $n_{e}^{\varepsilon}$ and of a single species of positively charged ions with density $n_{i}^{\varepsilon}$. Denote by $\phi^{\varepsilon}$ the electrostatic potential. These variables satisfy the scaled driftdiffusion equations (DD-EI):

$$
\begin{gathered}
\partial_{t} n_{i}^{\varepsilon}-\operatorname{div}\left(\nabla p_{i}\left(n_{i}^{\varepsilon}\right)+\mu_{i} n_{i}^{\varepsilon} \nabla \phi^{\varepsilon}\right)=0 \\
\partial_{t} n_{e}^{\varepsilon}-\operatorname{div}\left(\nabla p_{e}\left(n_{e}^{\varepsilon}\right)-\mu_{e} n_{e}^{\varepsilon} \nabla \phi^{\varepsilon}\right)=0 \\
-\varepsilon^{2} \Delta \phi^{\varepsilon}=n_{i}^{\varepsilon}-n_{e}^{\varepsilon}
\end{gathered}
$$

in $\Omega_{T}=(0, T) \times \Omega$, where $T>0$ and $\Omega \subset \mathbb{R}^{d}(d \geq 1)$ is an open and bounded domain. Here, $p_{i}$ and $p_{e}$ denote the pressure functions of the electrons and ions, respectively. The physical parameters are the (constant) mobilities $\mu_{i}>0, \mu_{e}>0$ and the (scaled) Debye length $\varepsilon>0$. We suppose that the boundary $\partial \Omega$ of the domain consists of two disjoint sets $\Gamma_{D}$ and $\Gamma_{N}$ with $\operatorname{meas}_{d-1}\left(\Gamma_{D}\right)>0$, and we denote by

$$
\Sigma_{D}=(0, T) \times \Gamma_{D}, \quad \Sigma_{N}=(0, T) \times \Gamma_{N} .
$$

The system (DD-EI) is complemented by initial conditions and mixed Dirichlet-Neumann boundary conditions for $\alpha=i, e$ :

$$
\begin{array}{cl}
n_{\alpha}^{\varepsilon}(0)=n_{I, \alpha} & \text { in } \Omega \\
n_{\alpha}^{\varepsilon}=n_{D, \alpha}, \quad \phi^{\varepsilon}=\phi_{D} & \text { on } \Sigma_{D} \\
\nabla p_{\alpha}\left(n_{\alpha}^{\varepsilon}\right) \cdot \nu=\nabla \phi^{\varepsilon} \cdot \nu=0 & \text { on } \Sigma_{N}
\end{array}
$$

Here the function $\nu$ is the normal unit vector of $\partial \Omega$ which is assumed to exist almost everywhere. We say that the boundary conditions are compatible on $\Sigma_{D}$ if

$$
n_{D, i}=n_{D, e} \text { on } \Sigma_{D}
$$

The quasi-neutral limit $\varepsilon \rightarrow 0$ is called also zero-Debye-length limit. For the drift-diffusion equations (1.1)-(1.6) this limit is justified in [14] under assumption that the electron and ion densities are equal at initial time, i.e. $n_{I, i}=n_{I, e}$, which avoids the formation of initial layer. However, the local compactness of the sequence $\left(n_{i}^{\varepsilon}, n_{e}^{\varepsilon}, \phi^{\varepsilon}\right)_{\varepsilon>0}$ is obtained without the compatibility condition (1.7). Since the sequences $\left(n_{\alpha}^{\varepsilon}\right)_{\varepsilon>0}$ converge to the same function for $\alpha=i$ and $\alpha=e$, we have in general boundary layers in the limiting process. We mention that this problem has also been studied by Gasser et al. [8,9] where assumption $p_{i}=p_{e}$ and homogeneous Neumann boundary conditions are imposed to simplify the analysis, since no boundary layers are expected.

The quasi-neutral limit in macroscopic models for plasmas has been investigated by Brézis et al. in [3] and by Cordier et al. in [4]. In the first mentioned paper the limit $\varepsilon \rightarrow 0$ is considered for the nonlinear Poisson equation (the ion density being fixed). In the second article the authors study the quasi-neutral limit for the traveling wave solutions of the hydrodynamic equations for plasmas. This limit is rigorously proved by Cordier and Grenier in [5] for locally smooth solutions of the one dimensional compressible Euler-Poisson equations and by Brenier for the weak solutions of the Vlasov-Poisson equations [2]. Asymptotic expansions in powers of $\varepsilon$ to the stationary drift-diffusion equations for semiconductors are derived by Markowich et al. [16,17].

This paper is organized as follows. In the next section, we first recall the main results obtained in [14]. Then we show the uniqueness of the quasi-neutral limit, which is determined by a initial-boundary value problem to a system of two decoupled equations. Indeed, the locally strong convergence of the sequence $\left(n_{i}^{\varepsilon}, n_{e}^{\varepsilon}\right)_{\varepsilon>0}$ in $L_{\mathrm{loc}}^{2}\left(\Omega_{T}\right)$ and the locally weak convergence of the sequence $\left(\phi^{\varepsilon}\right)_{\varepsilon>0}$ in $L^{2}\left(0, T ; H_{\mathrm{loc}}^{1}(\Omega)\right)$ shown in [14] allow to pass to the limit in the system (DD-EI), and the Dirichlet boundary conditions are obtained from the 
$L^{2}\left(0, T ; H^{1}(\Omega)\right)$ estimates for quasi-Fermi potentials. Thus, numerical simulation can be performed for the limiting problem. Section 3 is devoted to the boundary layer analysis of this limit in one space dimension. With the same scaling $x \rightarrow x / \varepsilon$ near $x=0$ used in $[3,6]$, we establish the boundary layer equations and prove the existence and uniqueness of solutions with exponential decay to 0 as $\varepsilon \rightarrow 0$. We stress that this result is still true in several space dimensions if the boundary layers occur only near a hyperplane of dimension $d-1$ of the form $\left\{x_{i}=a \in \mathbb{R}, 1 \leq i \leq d\right\}$ (see Rem. 4.1.). For more general boundary, we refer to [6,16] for the related analysis in the case of smooth solutions to stationary equations. Finally, in section 4 we prove the convergence rate $O\left(\varepsilon^{\frac{1}{2}}\right)$ of the quasi-neutral limit $n_{i}^{\varepsilon}-n_{e}^{\varepsilon} \longrightarrow 0$ in $L^{2}\left(\Omega_{T}\right)$. Since it is valid for the whole domain $\Omega_{T}$, we deduce a globally strong convergence of the sequence $\left(n_{i}^{\varepsilon}, n_{e}^{\varepsilon}\right)_{\varepsilon>0}$ in $L^{2}\left(\Omega_{T}\right)$. Moreover, we show that this convergence rate is optimal by an example of stationary drift-diffusion equations in one space dimension.

It turns out that we are not able to prove the strong convergence of the sequence $\left(\phi^{\varepsilon}\right)_{\varepsilon>0}$ due to the lack of a priori estimate for $\left(\partial_{t} \phi^{\varepsilon}\right)_{\varepsilon>0}$. However, it is possible to obtain a convergence rate in $L^{2}\left(\Omega_{T}\right)$ for the quantity $n_{\alpha}^{\varepsilon}-n$ in one space dimension, where $n$ denotes the limit of $n_{\alpha}^{\varepsilon}$ for $\alpha=i, e$. For this purpose, some special techniques are needed. This problem will be discussed in forthcoming publications.

For the sake of completeness, in the last section we give without proof a similar characterization of the quasi-neutral limit and the boundary layer analysis for the nonlinear drift-diffusion equations derived from the zero-electron-mass limit [12] in the drift-diffusion equations (DD-EI), where the electron density is replaced by the Boltzmann-Maxwell type relation. In this situation, we show that both two sequences $\left(n_{i}^{\varepsilon}\right)_{\varepsilon>0}$ and $\left(\phi^{\varepsilon}\right)_{\varepsilon>0}$ converge strongly in $L^{2}\left(\Omega_{T}\right)$.

\section{Preliminaries}

In this section, we first recall the main results established in [14] and then show that the quasi-neutral limit to (1.1)-(1.6) can be uniquely determined and a global convergence result (with respect to the domain) is available. The limit is governed by a system of two decoupled equations with given initial and boundary conditions which have not been shown in [14]. To this end, we need the same hypotheses used in [14]. For $\alpha=i, e$ :

(H1) $n_{I, e}=n_{I, i}$ in $\Omega$;

(H2) $n_{I, \alpha} \in L^{\infty}(\Omega), n_{D, \alpha} \in C^{0}\left(\Sigma_{D}\right) \cap H^{1}\left(\Sigma_{D}\right), \phi_{D} \in H^{1}\left(0, T ; H^{1}\left(\Gamma_{D}\right)\right) \cap L^{\infty}\left(\Sigma_{D}\right)$;

(H3) there exist two constants $\underline{n}, \bar{n}>0$ such that

$$
\underline{n} \leq n_{I, \alpha} \leq \bar{n} \text { in } \Omega \text { and } \underline{n} \leq n_{D, \alpha} \leq \bar{n} \text { on } \Sigma_{D} ;
$$

(H4) $p_{\alpha} \in C^{2}([0,+\infty))$ is strictly increasing on $(0,+\infty)$;

(H5) the function $s \longrightarrow s H(s)$ is strictly convex on $(0,+\infty)$, where

$$
h_{\alpha}^{\prime}(s)=p_{\alpha}^{\prime}(s) / s, \quad s>0, \quad h_{\alpha}(1)=0, \quad H=\frac{1}{\mu_{i}} h_{i}+\frac{1}{\mu_{e}} h_{e} .
$$

Under the assumptions (H2)-(H4) it has been proved in [10] that there exists a solution $\left(n_{i}^{\varepsilon}, n_{e}^{\varepsilon}, \phi^{\varepsilon}\right)$ to the problem (1.1)-(1.6) satisfying equations (1.1)-(1.3) in the sense of the usual variational formulation, the initial condition (1.4) in the sense of $V^{*}$, and

$$
n_{i}^{\varepsilon}, n_{e}^{\varepsilon} \in H^{1}\left(0, T ; V^{*}\right) \cap L^{\infty}\left(\Omega_{T}\right) \cap L^{2}\left(0, T ; H^{1}(\Omega)\right), \quad \phi^{\varepsilon} \in L^{\infty}\left(0, T ; H^{1}(\Omega)\right),
$$

where $V^{*}$ is the dual space of

$$
V=\left\{u \in H^{1}(\Omega) ; u=0 \text { on } \Gamma_{D}\right\} .
$$

If in addition, the given boundary data are smooth and $\Gamma_{N}=\emptyset$, the solution is unique. 
To study the quasi-neutral limit to the problem (1.1)-(1.6), let us introduce the quasi-Fermi potentials:

$$
F_{i}^{\varepsilon}=h_{i}\left(n_{i}^{\varepsilon}\right)+\mu_{i} \phi^{\varepsilon}, \quad F_{e}^{\varepsilon}=h_{e}\left(n_{e}^{\varepsilon}\right)-\mu_{e} \phi^{\varepsilon},
$$

which will play an important role in the proofs. The results obtained in [14] may be summarized as follows.

Lemma 2.1. Assume (H1)-(H4). Then for $\alpha=i, e$, we have:

(i) for almost all $(t, x) \in \Omega_{T}, \underline{n} \leq n_{\alpha}^{\varepsilon}(t, x) \leq \bar{n}$;

(ii) the sequence $\left(F_{\alpha}^{\varepsilon}\right)_{\varepsilon>0}$ is bounded in $L^{2}\left(0, T ; H^{1}(\Omega)\right)$;

(iii) the sequence $\left(n_{\alpha}^{\varepsilon}\right)_{\varepsilon>0}$ is bounded in $H^{1}\left(0, T ; H^{-1}(\Omega)\right)$;

(iv) the sequence $\left(\varepsilon \phi^{\varepsilon}\right)_{\varepsilon>0}$ is bounded in $L^{\infty}\left(0, T ; H^{1}(\Omega)\right)$;

(v) the following convergence holds for a subsequence (not relabeled) of $\left(n_{\alpha}^{\varepsilon}, \phi^{\varepsilon}\right)_{\varepsilon>0}$ :

$$
n_{\alpha}^{\varepsilon} \longrightarrow n \text { in } L_{\mathrm{loc}}^{2}\left(\Omega_{T}\right) \text { strongly, } \phi^{\varepsilon} \longrightarrow \phi \text { in } L^{2}\left(0, T ; H_{\mathrm{loc}}^{1}(\Omega)\right) \text { weakly; }
$$

(vi) for any open and bounded domain $\omega$ such that $\bar{\omega} \subset \Omega$, we have

$$
\left\|n_{i}^{\varepsilon}-n_{e}^{\varepsilon}\right\|_{L^{2}\left(\omega_{T}\right)} \leq A_{0} \varepsilon, \quad\left\|\nabla \phi^{\varepsilon}\right\|_{L^{2}\left(\omega_{T}\right)} \leq A_{0} \varepsilon
$$

where $\omega_{T}=(0, T) \times \omega$ and $A_{0}>0$ is a constant depending on $\omega$ and independent of $\varepsilon$. If in addition, the compatibility conditions (1.7) is satisfied, then the estimate (2.4) holds in the whole domain $\Omega_{T}$.

The assertion (i) is obtained from the maximum principle, and (ii) follows from the entropy type estimates which, together with the equations (1.1)-(1.2), implies (iii). The convergence (v) is a consequence of Aubin's Lemma [1,15] and the local estimates for the sequence $\left(n_{i}^{\varepsilon}, n_{e}^{\varepsilon}, \phi^{\varepsilon}\right)_{\varepsilon>0}$. Finally, the local convergence rate (2.4) is derived from a truncating argument. Note that in general this estimate cannot hold in the whole domain $\Omega_{T}$ due to the boundary layers. A global and optimal convergence rate weaker than (2.4) will be established in section 4 in one space dimension. The initial compatibility condition (H1) is needed only in showing the bounds of $\left(F_{\alpha}^{\varepsilon}\right)_{\varepsilon>0}$ in $L^{2}\left(0, T ; H^{1}(\Omega)\right)$. The quasi-neutral limit is an open problem if (H1) is not satisfied. We refer to $[8,9]$ for an initial layer analysis of the problem where new scalings are introduced.

From (ii) it is easy to see that $\left(\phi^{\varepsilon}\right)_{\varepsilon>0}$ is bounded in $L^{2}\left(\Omega_{T}\right)$. Therefore, $\phi \in L^{2}\left(\Omega_{T}\right)$ and from (i) $n \in L^{\infty}\left(\Omega_{T}\right)$ with $\underline{n} \leq n \leq \bar{n}$ in $\Omega_{T}$. Hence, there is a unique solution $\chi \in L^{\infty}\left(0, T ; H^{1}(\Omega)\right)$ to the following problem:

$$
\begin{gathered}
-\operatorname{div}(n \nabla \chi)=0 \text { in } \Omega_{T}, \\
\chi=\chi_{D} \quad \text { on } \Sigma_{D}, \quad \nabla \chi \cdot \nu=0 \text { on } \Sigma_{N},
\end{gathered}
$$

where

$$
\chi_{D}=h_{i}\left(n_{D, i}\right)-h_{e}\left(n_{D, e}\right)+\left(\mu_{i}+\mu_{e}\right) \phi_{D} .
$$

The main result of this section can be stated as follows. It shows that the quasi-neutral limit is unique and characterized by a initial boundary value problem to a decoupled system.

Theorem 2.1. Let the hypotheses (H1)-(H4) hold. Then the whole sequence $\left(n_{i}^{\varepsilon}, n_{e}^{\varepsilon}, \phi^{\varepsilon}\right)_{\varepsilon>0}$ converges to $(n, n, \phi)$ in the sense of (2.3). Moreover,

$$
n \in H^{1}\left(0, T ; H^{-1}(\Omega)\right) \cap L^{2}\left(0, T ; H^{1}(\Omega)\right) \cap L^{\infty}\left(\Omega_{T}\right), \quad \phi \in L^{2}\left(0, T ; H^{1}(\Omega)\right),
$$


and the pair $(n, \phi)$ is the unique solution of the following problem:

$$
\begin{array}{ll}
\partial_{t} n-\Delta p(n)=0 & \text { in } \Omega_{T}, \\
\phi=\frac{1}{\mu_{i}+\mu_{e}}\left(\chi+h_{e}(n)-h_{i}(n)\right) & \text { in } \Omega_{T}, \\
n(0)=n_{I, i} & \text { in } \Omega, \\
n=n_{D} \quad \text { on } \Sigma_{D}, \quad \nabla n \cdot \nu=0 & \text { on } \Sigma_{N},
\end{array}
$$

where

$$
p(n)=\frac{\mu_{e}}{\mu_{i}+\mu_{e}} p_{i}(n)+\frac{\mu_{i}}{\mu_{i}+\mu_{e}} p_{e}(n),
$$

and $n_{D}$ is uniquely determined by the relation

$$
H\left(n_{D}\right)=\frac{1}{\mu_{i}} h_{i}\left(n_{D, i}\right)+\frac{1}{\mu_{e}} h_{e}\left(n_{D, e}\right) .
$$

Proof. The inclusion $n \in H^{1}\left(0, T ; H^{-1}(\Omega)\right)$ follows from (iii) of Lemma 2.1. Therefore, (2.11) is satisfied in the sense of $H^{-1}(\Omega)$. To prove $n \in L^{2}\left(0, T ; H^{1}(\Omega)\right)$, we use the relation

$$
\frac{1}{\mu_{i}} F_{i}^{\varepsilon}+\frac{1}{\mu_{e}} F_{e}^{\varepsilon}=\frac{1}{\mu_{i}} h_{i}\left(n_{i}^{\varepsilon}\right)+\frac{1}{\mu_{e}} h_{e}\left(n_{e}^{\varepsilon}\right) .
$$

By (2.3), we have (up to a subsequence),

$$
\frac{1}{\mu_{i}} h_{i}\left(n_{i}^{\varepsilon}\right)+\frac{1}{\mu_{e}} h_{e}\left(n_{e}^{\varepsilon}\right) \longrightarrow \frac{1}{\mu_{i}} h_{i}(n)+\frac{1}{\mu_{e}} h_{e}(n)=H(n) \text { in } L_{\mathrm{loc}}^{2}\left(\Omega_{T}\right) \text { strongly. }
$$

Therefore, we obtain from (ii) of Lemma 2.1 that $H(n) \in L^{2}\left(0, T ; H^{1}(\Omega)\right)$. Since $h_{i}$ and $h_{e}$ are strictly increasing, so is $H$. By the $L^{\infty}\left(\Omega_{T}\right)$ bound for $n$, we have $n \in L^{2}\left(0, T ; H^{1}(\Omega)\right)$. This proves the first relation in (2.8).

Next, the local convergence (2.3) allows to pass to the limit in the system (DD-EI) in the sense of distributions to obtain

$$
\begin{aligned}
& \partial_{t} n-\operatorname{div}\left(\nabla p_{i}(n)+\mu_{i} n \nabla \phi\right)=0 \\
& \partial_{t} n-\operatorname{div}\left(\nabla p_{e}(n)-\mu_{e} n \nabla \phi\right)=0
\end{aligned}
$$

Adding and subtracting these two equations leads to (2.9) and

$$
-\operatorname{div}\left[\left(\mu_{i}+\mu_{e}\right) n \nabla \phi+\nabla p_{i}(n)-\nabla p_{e}(n)\right]=0,
$$

which can be rewritten as:

$$
-\operatorname{div}\left[n \nabla\left(\left(\mu_{i}+\mu_{e}\right) \phi+h_{i}(n)-h_{e}(n)\right)\right]=0 .
$$

Again from the limit $(2.3)$ and the $L^{2}\left(0, T ; H^{1}(\Omega)\right)$ bound for $\left(F_{i}^{\varepsilon}\right)_{\varepsilon>0}$, we deduce that

$$
F_{i}^{\varepsilon}=h_{i}\left(n_{i}^{\varepsilon}\right)+\mu_{i} \phi^{\varepsilon} \longrightarrow h_{i}(n)+\mu_{i} \phi \quad \text { in } L^{2}\left(0, T ; H^{1}(\Omega)\right) \text { weakly. }
$$

Therefore,

$$
\left.\left.\left(h_{i}\left(n_{i}^{\varepsilon}\right)+\mu_{i} \phi^{\varepsilon}\right)\right|_{\Sigma_{D}} \longrightarrow\left(h_{i}(n)+\mu_{i} \phi\right)\right|_{\Sigma_{D}} \quad \text { in } L^{2}\left(0, T ; H^{1 / 2}\left(\Gamma_{D}\right)\right) \text { weakly. }
$$


In view of the boundary conditions (1.5), we have

$$
h_{i}(n)+\mu_{i} \phi=h_{i}\left(n_{D, i}\right)+\mu_{i} \phi_{D} \text { on } \Sigma_{D} .
$$

In a same way, we have also

$$
h_{e}(n)-\mu_{e} \phi=h_{e}\left(n_{D, e}\right)-\mu_{e} \phi_{D} \quad \text { on } \Sigma_{D} .
$$

These two relations imply the Dirichlet boundary condition in (2.7) and (2.12). From the uniqueness of solutions to $(2.5)-(2.7)$, we obtain (2.10) which yields $\phi \in L^{2}\left(0, T ; H^{1}(\Omega)\right)$.

The uniqueness of solutions to the nonlinear heat equation (2.9) follows from the monotonicity of the function $p$, which yields the uniqueness of solutions to the problem (2.9)-(2.14). Finally, this uniqueness of solutions implies the convergence of the whole sequence $\left(n_{i}^{\varepsilon}, n_{e}^{\varepsilon}, \phi^{\varepsilon}\right)_{\varepsilon>0}$. This finishes the proof.

Theorem 2.1 gives a precise characterization of the limit $\varepsilon \rightarrow 0$ to the problem (1.1)-(1.6). Note that since the boundary layers exist, the sequence $\left(n_{i}^{\varepsilon}, n_{e}^{\varepsilon}, \phi^{\varepsilon}\right)_{\varepsilon>0}$ cannot be bounded in $L^{2}\left(0, T ; H^{1}(\Omega)\right)$. Therefore, $(2.8)$ is a regularity property of this limit. The following result concerns the globally strong convergence of the sequence $\left(n_{i}^{\varepsilon}, n_{e}^{\varepsilon}\right)_{\varepsilon>0}$, where the assumption (2.17) below will be proved in section 4 in one space dimension.

Proposition 2.1. Assume (H1)-(H5) hold and

$$
n_{i}^{\varepsilon}-n_{e}^{\varepsilon} \longrightarrow 0 \quad \text { in } L^{2}\left(\Omega_{T}\right) \text { strongly. }
$$

Then for $\alpha=i, e$,

$$
n_{\alpha}^{\varepsilon} \longrightarrow n \quad \text { in } L^{2}\left(\Omega_{T}\right) \text { strongly . }
$$

Proof. From (2.17) and the $L^{\infty}\left(\Omega_{T}\right)$ bounds for $\left(n_{\alpha}^{\varepsilon}\right)_{\varepsilon>0}$, we obtain for $\alpha=i, e$

$$
h_{\alpha}\left(n_{i}^{\varepsilon}\right)-h_{\alpha}\left(n_{e}^{\varepsilon}\right) \longrightarrow 0 \quad \text { in } L^{2}\left(\Omega_{T}\right) .
$$

Since $\left(n_{\alpha}^{\varepsilon}\right)_{\varepsilon>0}$ is bounded in $L^{\infty}\left(\Omega_{T}\right),\left(F_{\alpha}^{\varepsilon}\right)_{\varepsilon>0}$ is bounded in $L^{2}\left(0, T ; H^{1}(\Omega)\right)$ and

$$
\partial_{t} n_{\alpha}^{\varepsilon}-\operatorname{div}\left(n_{\alpha}^{\varepsilon} \nabla F_{\alpha}^{\varepsilon}\right)=0,
$$

by the div-curl lemma of compensated compactness [20] applied to the sequences $\left(n_{\alpha}^{\varepsilon}, n_{\alpha}^{\varepsilon} \nabla F_{\alpha}^{\varepsilon}\right)^{t}$ and $\left(F_{\alpha}^{\varepsilon}, 0\right)^{t}$, we have in the sense of distributions

$$
\lim _{\varepsilon \rightarrow 0} n_{\alpha}^{\varepsilon} F_{\alpha}^{\varepsilon}=\lim _{\varepsilon \rightarrow 0} n_{\alpha}^{\varepsilon} \lim _{\varepsilon \rightarrow 0} F_{\alpha}^{\varepsilon}, \quad \alpha=i, e,
$$

or equivalently,

$$
\lim _{\varepsilon \rightarrow 0} n_{i}^{\varepsilon}\left(h_{i}\left(n_{i}^{\varepsilon}\right)+\mu_{i} \phi^{\varepsilon}\right)=\lim _{\varepsilon \rightarrow 0} n_{i}^{\varepsilon} \lim _{\varepsilon \rightarrow 0}\left(h_{i}\left(n_{i}^{\varepsilon}\right)+\mu_{i} \phi^{\varepsilon}\right) \quad \text { in } D^{\prime}\left(\Omega_{T}\right)
$$

and

$$
\lim _{\varepsilon \rightarrow 0} n_{e}^{\varepsilon}\left(h_{e}\left(n_{e}^{\varepsilon}\right)-\mu_{e} \phi^{\varepsilon}\right)=\lim _{\varepsilon \rightarrow 0} n_{e}^{\varepsilon} \lim _{\varepsilon \rightarrow 0}\left(h_{e}\left(n_{e}^{\varepsilon}\right)-\mu_{e} \phi^{\varepsilon}\right) \quad \text { in } D^{\prime}\left(\Omega_{T}\right) .
$$

It follows from (2.17)-(2.18) that

$$
\lim _{\varepsilon \rightarrow 0} n_{e}^{\varepsilon} H\left(n_{e}^{\varepsilon}\right)=\lim _{\varepsilon \rightarrow 0} n_{e}^{\varepsilon} \lim _{\varepsilon \rightarrow 0} H\left(n_{e}^{\varepsilon}\right) \quad \text { in } D^{\prime}\left(\Omega_{T}\right) .
$$


Since $H$ is strictly increasing, from the monotonicity argument and the $L^{\infty}\left(\Omega_{T}\right)$ bound for $\left(n_{e}^{\varepsilon}\right)_{\varepsilon>0}$, we have the identification [15]

$$
\lim _{\varepsilon \rightarrow 0} H\left(n_{e}^{\varepsilon}\right)=H(n) \quad \text { in } L^{\infty}\left(\Omega_{T}\right) \text { weakly-*, }
$$

which yields

$$
\lim _{\varepsilon \rightarrow 0} n_{e}^{\varepsilon} H\left(n_{e}^{\varepsilon}\right)=n H(n) \quad \text { in } L^{\infty}\left(\Omega_{T}\right) \text { weakly-*. }
$$

Finally, we conclude the strong convergence of $\left(n_{e}^{\varepsilon}\right)_{\varepsilon>0}$ in $L^{2}\left(\Omega_{T}\right)$ from the strict convexity of the function $s \rightarrow s H(s)$, see [21]. The strong convergence of $\left(n_{i}^{\varepsilon}\right)_{\varepsilon>0}$ in $L^{2}\left(\Omega_{T}\right)$ follows immediately.

\section{BOUNDARY LAYER ANALYSIS}

In this section, we give a boundary layer analysis of the drift-diffusion equations in the above quasi-neutral limit. To avoid tedious computations, we only consider the problem in one space dimension. From now on, we denote by

$$
\Omega=(0,1), \quad \Omega_{T}=(0, T) \times(0,1), \quad \Gamma_{0}=\{x=0\}, \quad \Gamma_{1}=\{x=1\} .
$$

We suppose that only Dirichlet boundary conditions are prescribed. Therefore, the boundary layers may occur near $\Gamma_{0}$ and $\Gamma_{1}$. The situation is evidently simpler if a Neumann boundary condition is prescribed on $\Gamma_{0}$ or $\Gamma_{1}$.

In a neighborhood of $\Gamma_{0}$ and for $\alpha=i, e$, the solution $\left(n_{\alpha}^{\varepsilon}, \phi^{\varepsilon}\right)$ of (1.1)-(1.6) may be approximated by $(n(t, 0)+$ $\left.u_{\alpha}(t, y), \phi(t, 0)+\varphi(t, y)\right)$, where $y=x / \varepsilon$ is the fast variable. We expect that $\left(u_{i}(t, y), u_{e}(t, y), \varphi(t, y)\right)$ describes the boundary layer near $\Gamma_{0}$ in the quasi-neutral limit. Due to $(2.8)$ and Sobolev's imbedding $H^{1}(0,1) \hookrightarrow$ $C([0,1])$, the quantities $n(t, 0)$ and $\phi(t, 0)$ are well defined. Moreover, it is easy to see that $n(., 0)$ and $\phi(., 0)$ are continuous functions on $[0, T]$. Indeed, by $(2.15)-(2.16), n(t, 0)$ and $\phi(t, 0)$ can be expressed as

$$
\begin{aligned}
H(n(t, 0)) & =\frac{1}{\mu_{i}} h_{i}\left(n_{D, i}(t, 0)\right)+\frac{1}{\mu_{e}} h_{e}\left(n_{D, e}(t, 0)\right), \\
\phi(t, 0) & =\frac{1}{\mu_{i}}\left[h_{i}\left(n_{D, i}(t, 0)\right)-h_{i}(n(t, 0))+\mu_{i} \phi_{D}(t, 0)\right],
\end{aligned}
$$

and we obtain the continuity of $n(t, 0)$ and $\phi(t, 0)$ from the assumptions (H2) and (H4).

Now we establish boundary layer equations for $\left(u_{i}(t, y), u_{e}(t, y), \varphi(t, y)\right)$. Putting the approximate solution $\left(n(t, 0)+u_{i}(t, y), n(t, 0)+u_{e}(t, y), \phi(t, 0)+\varphi(t, y)\right)$ into the drift-diffusion equations (DD-EI) and taking into account the $\varepsilon^{-2}$ term in (1.1)-(1.2) and the $\varepsilon^{0}$ term in (1.3), we obtain:

$$
\begin{gathered}
\partial_{y}\left[\left(n(t, 0)+u_{i}(t, y)\right) \partial_{y}\left(h_{i}\left(n(t, 0)+u_{i}(t, y)\right)+\mu_{i} \varphi(t, y)\right)\right]=0 \\
\partial_{y}\left[\left(n(t, 0)+u_{e}(t, y)\right) \partial_{y}\left(h_{e}\left(n(t, 0)+u_{e}(t, y)\right)-\mu_{e} \varphi(t, y)\right)\right]=0 \\
-\partial_{y y} \varphi(t, y)=u_{i}(t, y)-u_{e}(t, y)
\end{gathered}
$$

for $(t, y) \in B_{T}:=(0, T) \times(0,+\infty)$. The boundary conditions of these variables are given by, for $\alpha=i, e$,

$$
\begin{array}{lrl}
u_{\alpha}(t, 0)=n_{D, \alpha}(t, 0)-n(t, 0), & \lim _{y \rightarrow+\infty} u_{\alpha}(t, y)=0 & \text { in }[0, T] \\
\varphi(t, 0)=\phi_{D}(t, 0)-\phi(t, 0):=\varphi_{b l}(t), & \lim _{y \rightarrow+\infty} \varphi(t, y)=0 & \text { in }[0, T]
\end{array}
$$


Now we simplify the above equations. Integrating (3.1)-(3.2) and using the boundary conditions (3.4)-(3.5), we obtain

$$
\begin{aligned}
h_{i}\left(n(t, 0)+u_{i}(t, y)\right)+\mu_{i} \varphi(t, y) & =h_{i}\left(n_{D, i}(t, 0)\right)+\mu_{i} \varphi_{b l}(t):=a_{i}(t) \\
h_{e}\left(n(t, 0)+u_{e}(t, y)\right)-\mu_{e} \varphi(t, y) & =h_{e}\left(n_{D, e}(t, 0)\right)-\mu_{e} \varphi_{b l}(t):=a_{e}(t)
\end{aligned}
$$

From the definition of $\varphi_{b l}$ and (2.15)-(2.16), we have also

$$
a_{\alpha}(t)=h_{\alpha}(n(t, 0)), \quad \alpha=i, e .
$$

Let $f_{\alpha}$ be the inverse function of $h_{\alpha}(\alpha=i, e)$. It is obvious that $f_{\alpha} \in C^{1}$. Then we obtain from the above equations:

$$
\begin{array}{ll}
u_{i}(t, y)=f_{i}\left(a_{i}(t)-\mu_{i} \varphi(t, y)\right)-f_{i}\left(a_{i}(t)\right) & \text { in } B_{T} \\
u_{e}(t, y)=f_{e}\left(a_{e}(t)+\mu_{e} \varphi(t, y)\right)-f_{e}\left(a_{e}(t)\right) & \text { in } B_{T}
\end{array}
$$

and then from $(3.3)$

$$
\partial_{y y} \varphi(t, y)=f_{e}\left(a_{e}(t)+\mu_{e} \varphi(t, y)\right)-f_{i}\left(a_{i}(t)-\mu_{i} \varphi(t, y)\right) \text { in } B_{T}
$$

Similarly, in a neighborhood of $\Gamma_{1}$ and for $\alpha=i, e$, the solution $\left(n_{\alpha}^{\varepsilon}, \phi^{\varepsilon}\right)$ may be approximated by $(n(t, 1)+$ $\left.v_{\alpha}(t, z), \phi(t, 1)+\psi(t, z)\right)$, where $z=(1-x) / \varepsilon$. The variables $\left(v_{i}(t, z), v_{e}(t, z), \psi(t, z)\right)$ satisfy the boundary layer equations:

$$
\begin{aligned}
v_{i}(t, z) & =f_{i}\left(b_{i}(t)-\mu_{i} \psi(t, z)\right)-f_{i}\left(b_{i}(t)\right) & & \text { in } B_{T}, \\
v_{e}(t, z) & =f_{e}\left(b_{e}(t)+\mu_{e} \psi(t, z)\right)-f_{e}\left(b_{e}(t)\right) & & \text { in } B_{T}, \\
\partial_{z z} \psi(t, z) & =f_{e}\left(b_{e}(t)+\mu_{e} \psi(t, z)\right)-f_{i}\left(b_{i}(t)-\mu_{i} \psi(t, z)\right) & & \text { in } B_{T},
\end{aligned}
$$

with boundary conditions:

$$
\begin{array}{lll}
v_{\alpha}(t, 1)=n_{D, \alpha}(t, 1)-n(t, 1), & \lim _{z \rightarrow+\infty} v_{\alpha}(t, z)=0 & \text { in }[0, T], \\
\psi(t, 1)=\phi_{D}(t, 1)-\phi(t, 1):=\psi_{b l}(t), & \lim _{z \rightarrow+\infty} \psi(t, z)=0 & \text { in }[0, T],
\end{array}
$$

where

$$
b_{\alpha}(t)=h_{\alpha}(n(t, 1)), \quad \alpha=i, e .
$$

Notice that the boundary conditions (3.4)-(3.5) and (3.13)-(3.14) are meaningful due to (H2) and (2.8). Obviously, for $\alpha=i, e, a_{\alpha}, b_{\alpha}, \varphi_{b l}$ and $\psi_{b l}$ are given continuous functions on $[0, T]$.

The following result shows the existence and uniqueness of the boundary layers $\left(u_{i}, u_{e}, \varphi\right)$ and $\left(v_{i}, v_{e}, \psi\right)$ with exponential decay to 0 as $y \rightarrow+\infty$ and $z \rightarrow+\infty$, respectively.

Theorem 3.1. Assume (H1)-(H4) hold. Then for all $t \in[0, T]$, the boundary layer equations (3.7)-(3.9) and (3.5) ((3.10)-(3.12) and (3.14) respectively) admit a unique solution $\left(u_{i}, u_{e}, \varphi\right)\left(\left(v_{i}, v_{e}, \psi\right)\right.$ respectively), which is continuous in $t$, of class $C^{3}$ and monotone in $y$ (z respectively), such that for $\alpha=i, e$ :

$$
\begin{gathered}
\left|u_{\alpha}(t, y)\right|,|\varphi(t, y)| \leq C_{\varphi}\left|\varphi_{b l}(t)\right| \exp \left(-\delta_{\varphi} y\right), \quad(t, y) \in[0, T] \times(0,+\infty), \\
\left|v_{\alpha}(t, z)\right|,|\psi(t, z)| \leq C_{\psi}\left|\psi_{b l}(t)\right| \exp \left(-\delta_{\psi} z\right), \quad(t, z) \in[0, T] \times(0,+\infty)
\end{gathered}
$$


Moreover,

$$
\begin{aligned}
& \left|\partial_{y} u_{\alpha}(t, y)\right|,\left|\partial_{y} \varphi(t, y)\right| \leq C_{\varphi}\left|\varphi_{b l}(t)\right| \exp \left(-\delta_{\varphi} y\right), \quad(t, y) \in[0, T] \times(0,+\infty) \\
& \left|\partial_{z} v_{\alpha}(t, z)\right|,\left|\partial_{z} \psi(t, z)\right| \leq C_{\psi}\left|\psi_{b l}(t)\right| \exp \left(-\delta_{\psi} z\right), \quad(t, z) \in[0, T] \times(0,+\infty)
\end{aligned}
$$

where $C_{\varphi}>0$ and $\delta_{\varphi}>0\left(C_{\psi}>0\right.$ and $\delta_{\psi}>0$ respectively) are two constants depending only on $T$ and $\varphi_{b l}\left(\psi_{b l}\right.$ respectively).

Proof. Let us define the function $f$ by:

$$
f(t, \varphi)=f_{e}\left(a_{e}(t)+\mu_{e} \varphi\right)-f_{i}\left(a_{i}(t)-\mu_{i} \varphi\right), \quad(t, \varphi) \in[0, T] \times \mathbb{R} .
$$

It is clear that $f$ is continuous in $t$ and of class $C^{1}$ in $\varphi$. Since $p_{i}$ and $p_{e}$ are strictly increasing, so are $f_{i}$ and $f_{e}$. We obtain for any $(t, \varphi) \in[0, T] \times \mathbb{R}$,

$$
\frac{\partial f(t, \varphi)}{\partial \varphi}=\mu_{e} f_{e}^{\prime}\left(a_{e}(t)+\mu_{e} \varphi\right)+\mu_{i} f_{i}^{\prime}\left(a_{i}(t)-\mu_{i} \varphi\right)>0
$$

and there is a constant $f_{0}>0$ depending only on $T$ and $\varphi_{b l}$, such that

$$
\frac{\partial f(t, \varphi)}{\partial \varphi} \geq f_{0}, \quad \text { for all }(t, \varphi) \in[0, T] \times\left[-\left|\varphi_{b l}(t)\right|,\left|\varphi_{b l}(t)\right|\right]
$$

From

$$
f(t, 0)=f_{e}\left(a_{e}(t)\right)-f_{i}\left(a_{i}(t)\right)=n(t, 0)-n(t, 0)=0,
$$

we deduce that

$$
f(t, \varphi)>0 \text { for } \varphi>0, \quad f(t, \varphi)<0 \text { for } \varphi<0 .
$$

For $t \in[0, T]$ such that $\varphi_{b l}(t)>0$, the following problem

$$
\partial_{y y} \varphi(t, y)=f(t, \varphi), \quad \varphi(t, 0)=\varphi_{b l}(t), \quad \lim _{y \rightarrow+\infty} \varphi(t, y)=0
$$

admits a unique solution $\varphi(t, y)$ which satisfies (see [6], Lemma 2.1):

$$
y=\int_{\varphi}^{\varphi_{b l}(t)} \frac{\mathrm{d} s}{\sqrt{2 F(t, s)}},
$$

where

$$
F(t, s)=\int_{0}^{s} f(t, y) \mathrm{d} y
$$

Obviously, this solution is monotone decreasing in $y$. Therefore,

$$
0 \leq \varphi(t, y) \leq \varphi_{b l}(t), \quad \forall y \in(0,+\infty)
$$

From

$$
F(t, 0)=0, \quad \frac{\partial F(t, 0)}{\partial s}=f(t, 0)=0,
$$


we obtain for some $\xi \in[0, s]$,

$$
F(t, s)=\frac{\partial f(t, \xi)}{\partial s} s^{2} / 2 \geq f_{0} s^{2} / 2, \quad \forall s \in\left[0, \varphi_{b l}(t)\right] .
$$

Hence,

$$
0 \leq y \leq \int_{\varphi}^{\varphi_{b l}(t)} \frac{\mathrm{d} s}{\sqrt{f_{0}} s}=\frac{1}{\sqrt{f_{0}}} \log \left(\frac{\varphi_{b l}(t)}{\varphi}\right)
$$

and then

$$
0 \leq \varphi \leq \varphi_{b l}(t) \exp \left(-\sqrt{f_{0}} y\right)
$$

The estimate for $\partial_{y} \varphi$ follows from the relations:

$$
-\partial_{y} \varphi=\sqrt{2 F(t, \varphi)}, \quad F(t, 0)=0 .
$$

For $t \in[0, T]$ such that $\varphi_{b l}(t)<0$, we may replace $\varphi$ by $\bar{\varphi}=-\varphi$, which satisfies

$$
\begin{gathered}
\partial_{y y} \bar{\varphi}(t, y)=\bar{f}(t, \bar{\varphi}):=-f(t,-\bar{\varphi}), \\
\bar{\varphi}(t, 0)=-\varphi_{b l}(t)>0, \quad \lim _{y \rightarrow+\infty} \bar{\varphi}(t, y)=0 .
\end{gathered}
$$

Since

$$
\bar{f}(t, 0)=0, \quad \frac{\partial \bar{f}(t, \bar{\varphi})}{\partial \bar{\varphi}}=\frac{\partial f(t, \varphi)}{\partial \varphi}>0, \quad \forall(t, \bar{\varphi}) \in[0, T] \times \mathbb{R},
$$

the above result can be applied to $\bar{\varphi}$. In this case, $\varphi(t, y)$ is monotone increasing in $y$ and we have

$$
\varphi_{b l}(t) \exp \left(-\sqrt{f_{0}} y\right) \leq \varphi(t, y) \leq 0, \quad \forall y \in(0,+\infty) .
$$

The case where $\varphi_{b l}(t)=0$ is trivial. It leads to $\varphi(t, y)=0$. The same results for $u_{i}$ and $u_{e}$ follow from (3.7)-(3.8). Finally, the regularity of solutions is derived from the equations (3.9), (3.12) and $f_{\alpha} \in C^{1}$. The proof for $\left(v_{i}, v_{e}, \psi\right)$ is similar.

\section{Convergence Rate of $\left\|n_{i}^{\varepsilon}-n_{e}^{\varepsilon}\right\|_{L^{2}\left(\Omega_{T}\right)}$}

The results of the boundary layer analysis allow to establish a global convergence rate for $n_{i}^{\varepsilon}-n_{e}^{\varepsilon}$ with respect to the domain $\Omega$. This implies a strong convergence of the sequence $\left(n_{i}^{\varepsilon}, n_{e}^{\varepsilon}\right)_{\varepsilon>0}$ in the whole domain $\Omega_{T}$, which have not been provided in [14]. To see this, let's denote by

$$
R^{\varepsilon}(t, x)=n_{i}^{\varepsilon}(t, x)-u_{i}^{\varepsilon}(t, x)-v_{i}^{\varepsilon}(t, x)-\left[n_{e}^{\varepsilon}(t, x)-u_{e}^{\varepsilon}(t, x)-v_{e}^{\varepsilon}(t, x)\right]
$$

where

$$
u_{\alpha}^{\varepsilon}(t, x)=u_{\alpha}(t, y)=u_{\alpha}\left(t, \frac{x}{\varepsilon}\right), v_{\alpha}^{\varepsilon}(t, x)=v_{\alpha}(t, z)=v_{\alpha}\left(t, \frac{1-x}{\varepsilon}\right), \quad \alpha=i, e .
$$


The main idea is to use $R^{\varepsilon}$ instead of $n_{i}^{\varepsilon}-n_{e}^{\varepsilon}$ in the corresponding estimate. Indeed, from Theorem 3.1, it is easy to see that there exists a constant $C_{1}>0$ depending only on $T, \varphi_{b l}$ and $\psi_{b l}$ such that

$$
\left\|u_{\alpha}^{\varepsilon}(t, .)\right\|_{L^{2}(\Omega)} \leq C_{1} \varepsilon^{\frac{1}{2}},\left\|v_{\alpha}^{\varepsilon}(t, .)\right\|_{L^{2}(\Omega)} \leq C_{1} \varepsilon^{\frac{1}{2}}, \quad \alpha=i, e,
$$

and from (3.4) and (3.13), we have

$$
\begin{aligned}
& \left|R^{\varepsilon}(t, 0)\right|=\left|\left(v_{e}-v_{i}\right)(t, 1 / \varepsilon)\right| \leq 2 C_{\psi} \exp \left(-\delta_{\psi} / \varepsilon\right) \\
& \left|R^{\varepsilon}(t, 1)\right|=\left|\left(u_{e}-u_{i}\right)(t, 1 / \varepsilon)\right| \leq 2 C_{\varphi} \exp \left(-\delta_{\varphi} / \varepsilon\right)
\end{aligned}
$$

The key result to prove the global convergence rate of $n_{i}^{\varepsilon}-n_{e}^{\varepsilon}$ in $L^{2}\left(\Omega_{T}\right)$ is the following lemma. See Appendix for its proof.

Lemma 4.1. Under the assumptions (H1)-(H4), there is a constant $A_{1}>0$ independent of $\varepsilon$ such that

$$
\left\|R^{\varepsilon}\right\|_{L^{2}\left(\Omega_{T}\right)} \leq A_{1} \varepsilon^{\frac{1}{2}},\left\|\varepsilon^{\frac{1}{2}} \partial_{x} \phi^{\varepsilon}\right\|_{L^{2}\left(\Omega_{T}\right)} \leq A_{1}
$$

From the definition of $R^{\varepsilon}$, Proposition 2.1 and (4.1), we deduce the following global convergence rate for $n_{i}^{\varepsilon}-n_{e}^{\varepsilon}$ and the globally strong convergence of the sequence $\left(n_{i}^{\varepsilon}, n_{e}^{\varepsilon}\right)_{\varepsilon>0}$ in $L^{2}\left(\Omega_{T}\right)$.

Theorem 4.1. Under the assumptions of (H1)-(H5), there is a constant $A_{2}>0$ independent of $\varepsilon$ such that

$$
\left\|n_{i}^{\varepsilon}-n_{e}^{\varepsilon}\right\|_{L^{2}\left(\Omega_{T}\right)} \leq A_{2} \varepsilon^{\frac{1}{2}}
$$

Moreover, for $\alpha=i, e$, we have

$$
n_{i}^{\varepsilon} \longrightarrow n, n_{e}^{\varepsilon} \longrightarrow n \text { in } L^{2}\left(\Omega_{T}\right) \text { strongly }
$$

In order to show that the estimate (4.5) is optimal, let us consider the following stationary model of the drift-diffusion equations in one space dimension.

Example. Take $\mu_{i}=\mu_{e}=1$ and $p_{i}(s)=p_{e}(s)=s^{2} / 2$, and define $q_{i}=1$ and $q_{e}=-1$, then the stationary drift-diffusion equations are written as:

$$
\begin{gathered}
-\frac{\mathrm{d}}{\mathrm{d} x}\left[n_{\alpha}^{\varepsilon} \frac{\mathrm{d}}{\mathrm{d} x}\left(n_{\alpha}^{\varepsilon}+q_{\alpha} \phi^{\varepsilon}\right)\right]=0, \quad \alpha=i, e, \\
-\varepsilon^{2} \frac{\mathrm{d}^{2} \phi^{\varepsilon}}{\mathrm{d} x^{2}}=n_{i}^{\varepsilon}-n_{e}^{\varepsilon}
\end{gathered}
$$

in $\Omega=(0,1)$, subject to the boundary conditions

$$
\begin{aligned}
& n_{\alpha}^{\varepsilon}(0)=n_{\alpha}^{0}, \quad \phi^{\varepsilon}(0)=\phi^{0}, \quad \alpha=i, e \\
& n_{\alpha}^{\varepsilon}(1)=n_{\alpha}^{1}, \quad \phi^{\varepsilon}(1)=\phi^{1}, \quad \alpha=i, e
\end{aligned}
$$

We suppose that $n_{\alpha}^{0}>0$ and $n_{\alpha}^{1}>0$ for $\alpha=i, e$. Then it is easy to check that the solution of the above problem satisfies the following relations:

$$
n_{i}^{\varepsilon}+\phi^{\varepsilon}=\beta_{i}^{\varepsilon} \int_{0}^{x} \frac{\mathrm{d} y}{n_{i}^{\varepsilon}(y)}+n_{i}^{0}+\phi^{0}, \quad n_{e}^{\varepsilon}-\phi^{\varepsilon}=\beta_{e}^{\varepsilon} \int_{0}^{x} \frac{\mathrm{d} y}{n_{e}^{\varepsilon}(y)}+n_{e}^{0}-\phi^{0},
$$


where the constants $\beta_{i}^{\varepsilon}$ and $\beta_{e}^{\varepsilon}$ are determined by

$$
\beta_{i}^{\varepsilon} \int_{0}^{1} \frac{\mathrm{d} y}{n_{i}^{\varepsilon}(y)}+n_{i}^{0}+\phi^{0}=n_{i}^{1}+\phi^{1}, \quad \beta_{e}^{\varepsilon} \int_{0}^{1} \frac{\mathrm{d} y}{n_{e}^{\varepsilon}(y)}+n_{e}^{0}-\phi^{0}=n_{e}^{1}-\phi^{1} .
$$

Now we suppose furthermore that

$$
n_{i}^{0}+\phi^{0}=n_{i}^{1}+\phi^{1}, n_{e}^{0}-\phi^{0}=n_{e}^{1}-\phi^{1} .
$$

Hence,

$$
\beta_{i}^{\varepsilon}=\beta_{e}^{\varepsilon}=0
$$

Let us denote by

$$
d=n_{i}^{0}-n_{e}^{0}+2 \phi^{0}=n_{i}^{1}-n_{e}^{1}+2 \phi^{1},
$$

then we obtain from the Poisson equation

$$
-\varepsilon^{2} \frac{\mathrm{d}^{2} \phi^{\varepsilon}}{\mathrm{d} x^{2}}=d-2 \phi^{\varepsilon}
$$

whose solution is given explicitly by

$$
\phi^{\varepsilon}=A_{1}^{\varepsilon} \exp (\sqrt{2} x / \varepsilon)+A_{2}^{\varepsilon} \exp (-\sqrt{2} x / \varepsilon)+d / 2,
$$

where

$$
A_{1}^{\varepsilon}=\frac{\left(2 \phi^{1}-d\right)-\left(2 \phi^{0}-d\right) \exp (-\sqrt{2} / \varepsilon)}{2[\exp (\sqrt{2} / \varepsilon)-\exp (-\sqrt{2} / \varepsilon)]}, \quad A_{2}^{\varepsilon}=\frac{\left(2 \phi^{0}-d\right) \exp (\sqrt{2} / \varepsilon)-\left(2 \phi^{1}-d\right)}{2[\exp (\sqrt{2} / \varepsilon)-\exp (-\sqrt{2} / \varepsilon)]}
$$

Thus,

$$
\left\|n_{i}^{\varepsilon}-n_{e}^{\varepsilon}\right\|_{L^{2}(\Omega)}=\left\|2 \phi^{\varepsilon}-d\right\|_{L^{2}(\Omega)}=\left(\left|2 \phi^{1}-d\right|+\left|2 \phi^{0}-d\right|\right) O\left(\varepsilon^{\frac{1}{2}}\right) .
$$

It is clear that $2 \phi^{1}=2 \phi^{0}=d$ if and only if the compatibility condition (1.7) holds, i.e. $n_{i}^{0}=n_{e}^{0}$ and $n_{i}^{1}=n_{e}^{1}$. This shows that the convergence rate (4.5) is optimal.

In this example, since the limit $(n, \phi)$ of $\left(n_{\alpha}^{\varepsilon}, \phi^{\varepsilon}\right)$ is given by

$$
\phi=d / 2, \quad n=n_{i}^{0}+\phi^{0}-\phi,
$$

we have also:

$$
\left\|n_{\alpha}^{\varepsilon}-n\right\|_{L^{2}(\Omega)}=\left\|\phi^{\varepsilon}-\phi\right\|_{L^{2}(\Omega)}=\left(\left|2 \phi^{1}-d\right|+\left|2 \phi^{0}-d\right|\right) O\left(\varepsilon^{\frac{1}{2}}\right), \quad \alpha=i, e .
$$

We end this section by the following remark.

Remark 4.1. The results presented in Sections 3-4 are still true in several space dimensions if the boundary layer phenomenon occurs only near a hyperplane of dimension $d-1$ of the form $\Gamma_{a}=\left\{x_{i}=a \in \mathbb{R}, 1 \leq\right.$ $i \leq d\}$. To see this assertion, suppose that the domain $\Omega$ is located on the right of $\Gamma_{a}$. Then for $\alpha=i, e$, we way approach $\left(n_{\alpha}^{\varepsilon}, \phi^{\varepsilon}\right)$ by $\left(n\left(t, x^{\prime}, a\right)+u_{\alpha}\left(t, x^{\prime}, y\right), \phi\left(t, x^{\prime}, a\right)+\varphi\left(t, x^{\prime}, y\right)\right)$ in a neighborhood of $\Gamma_{a}$, where $x^{\prime}=\left(x_{1}, \ldots x_{i-1}, x_{i+1}, \ldots, x_{d}\right)$ and $y=\left(x_{i}-a\right) / \varepsilon$. Following the discussion in Section 3 , we obtain the same 
boundary layer equations for $\left(u_{\alpha}\left(t, x^{\prime}, y\right), \varphi\left(t, x^{\prime}, y\right)\right)$ and the existence and uniqueness of solutions. Moreover, for $x \in \Omega$ and $(t, y) \in[0, T] \times(0,+\infty)$, the following estimates hold:

$$
\begin{aligned}
\left|u_{\alpha}\left(t, x^{\prime}, y\right)\right|,\left|\varphi\left(t, x^{\prime}, y\right)\right| & \leq C_{\varphi}\left|\varphi_{b l}\left(t, x^{\prime}\right)\right| \exp \left(-\delta_{\varphi} y\right), \\
\left|\partial_{y} u_{\alpha}\left(t, x^{\prime}, y\right)\right|,\left|\partial_{y} \varphi\left(t, x^{\prime}, y\right)\right| & \leq C_{\varphi}\left|\varphi_{b l}\left(t, x^{\prime}\right)\right| \exp \left(-\delta_{\varphi} y\right),
\end{aligned}
$$

and

$$
\left|\partial_{x_{j}} u_{\alpha}\left(t, x^{\prime}, y\right)\right|,\left|\partial_{x_{j}} \varphi\left(t, x^{\prime}, y\right)\right| \leq C_{\varphi}\left|\varphi_{b l}\left(t, x^{\prime}\right)\right| \exp \left(-\delta_{\varphi} y\right), \quad j \neq i
$$

where $\varphi_{b l}\left(t, x^{\prime}\right)=\phi_{D}\left(t, x^{\prime}\right)-\phi\left(t, x^{\prime}, a\right)$. The constants $C_{\varphi}>0$ and $\delta_{\varphi}>0$ depend only on $T$ and $\varphi_{b l}$. Indeed, the last estimate above follows again from the boundary layer equations:

$$
\begin{aligned}
\partial_{y y} \varphi & =f(t, \varphi), \quad y \in(0,+\infty), \\
\varphi\left(t, x^{\prime}, 0\right) & =\varphi_{b l}\left(t, x^{\prime}\right), \quad \lim _{y \rightarrow+\infty} \varphi\left(t, x^{\prime}, y\right)=0,
\end{aligned}
$$

where $f$ is defined in (3.16). Indeed, using the above equations we obtain explicitly

$$
\partial_{x_{j}} \varphi=\frac{\sqrt{F(t, \varphi)}}{\sqrt{F\left(t, \varphi_{b l}\right)}} \partial_{x_{j}} \varphi_{b l},
$$

with

$$
F(t, \varphi)=\int_{0}^{\varphi} f(t, s) \mathrm{d} s .
$$

The remainder of the proof is similar to that of Section 4. We obtain Lemma 4.1 and Theorem 4.1 under assumption of some regularity conditions on $\varphi_{b l}$.

\section{BOUNDARY LAYER ANALYSIS IN THE NONLINEAR DRIFT-DIFFUSION EQUATIONS}

The nonlinear drift-diffusion equations are the system of equations (1.1) and (1.3) in which the electron density is replaced by:

$$
n_{e}^{\varepsilon}=f_{e}\left(\mu_{e} \phi^{\varepsilon}\right)
$$

which is a generalization of the Boltzmann-Maxwell relation $[3,18,19] n_{e}^{\varepsilon}=\exp \left(\phi^{\varepsilon}\right)$ obtained by the choice $p_{e}(s)=\mu_{e} s$. The nonlinear drift-diffusion equations are valid when the kinetic energy of the electrons is much smaller than their thermal energy. The justification of this asymptotic limit to the drift-diffusion equations can be found in [12]. In this situation, the ion density and the electrostatic potential solve the problem (DD-I):

$$
\begin{aligned}
\partial_{t} n^{\varepsilon}-\operatorname{div}\left(\nabla p_{i}\left(n^{\varepsilon}\right)+\mu_{i} n^{\varepsilon} \nabla \phi^{\varepsilon}\right)=0 & \text { in } \Omega_{T}, \\
-\varepsilon^{2} \Delta \phi^{\varepsilon}=n^{\varepsilon}-f_{e}\left(\mu_{e} \phi^{\varepsilon}\right) & \text { in } \Omega_{T},
\end{aligned}
$$

subject to the initial and boundary conditions

$$
\begin{aligned}
n^{\varepsilon}(0)=n_{I} & \text { in } \Omega \\
n^{\varepsilon}=n_{D}, \quad \phi^{\varepsilon}=\phi_{D} & \text { on } \Sigma_{D} \\
\nabla p_{i}\left(n^{\varepsilon}\right) \cdot \nu=\nabla \phi^{\varepsilon} \cdot \nu=0 & \text { on } \Sigma_{N}
\end{aligned}
$$

where we have used a simple notation $n^{\varepsilon}=n_{i}^{\varepsilon}$. 
By a straightforward computation, it is easy to see that the limit $(n, \phi)$ of $\left(n^{\varepsilon}, \phi^{\varepsilon}\right)$ as $\varepsilon \rightarrow 0$ satisfies the following equations:

$$
\begin{array}{rr}
\partial_{t} n-\frac{\mu_{i}+\mu_{e}}{\mu_{i} \mu_{e}} \Delta p(n)=0 & \text { in } \Omega_{T}, \\
\phi=h_{e}(n) / \mu_{e} & \text { in } \Omega_{T},
\end{array}
$$

where $p$ is defined in (2.13). This quasi-neutral limit has also been proved in [14]. Due to the nonlinear Poisson equation (5.2), we have a $L^{\infty}\left(\Omega_{T}\right)$ estimate for the sequence $\left(\phi^{\varepsilon}\right)_{\varepsilon>0}$, which yields a globally strong convergence of the sequences $\left(n^{\varepsilon}, \phi^{\varepsilon}\right)_{\varepsilon>0}$ in $L^{2}\left(\Omega_{T}\right)$ without the compatibility condition on the data given on $\Sigma_{D}$ :

$$
\phi_{D}=h_{e}\left(n_{D}\right) / \mu_{e} .
$$

Thus the boundary layers may exist too. The goal of this section is to give (without proof) a similar analysis of this boundary layer. We first state the result of the characterization of the limit.

Theorem 5.1. Let the hypotheses (H2)-(H3) for $\alpha=i$, (H4) for $\alpha=i, e$ and (H5) hold. Furthermore, $f_{e}(0)>0$ and $f_{e}(\infty)=+\infty$. Then the whole sequence $\left(n^{\varepsilon}, \phi^{\varepsilon}\right)_{\varepsilon>0}$ of solutions to (5.1)-(5.5) converges to $(n, \phi)$ in the following sense:

$$
\begin{aligned}
& n^{\varepsilon} \longrightarrow n \text { in } L^{2}\left(\Omega_{T}\right) \text { strongly, } \\
& \phi^{\varepsilon} \longrightarrow \phi \text { in } L^{2}\left(\Omega_{T}\right) \text { strongly and in } L^{2}\left(0, T ; H_{\mathrm{loc}}^{1}(\Omega)\right) \text { weakly. }
\end{aligned}
$$

The limit $(n, \phi)$ satisfies (2.8) and $\phi \in L^{\infty}\left(\Omega_{T}\right)$, and is the unique solution of the equations (5.6)-(5.7) with initial and boundary conditions:

$$
\begin{aligned}
n(0)=n_{I} & \text { in } \Omega, \\
n=\bar{n}_{D} & \text { on } \Sigma_{D}, \\
\nabla n \cdot \nu=\nabla \phi \cdot \nu=0 & \text { on } \Sigma_{N},
\end{aligned}
$$

where the Dirichlet data $\bar{n}_{D}$ is given by the relation:

$$
H\left(\bar{n}_{D}\right)=\frac{1}{\mu_{i}} h_{i}\left(n_{D}\right)+\phi_{D}
$$

Moreover, we have the optimal estimates:

$$
\left\|n^{\varepsilon}-f_{e}\left(\mu_{e} \phi^{\varepsilon}\right)\right\|_{L^{2}\left(\Omega_{T}\right)}=O\left(\varepsilon^{\frac{1}{2}}\right), \quad\left\|\nabla \phi^{\varepsilon}\right\|_{L^{2}\left(\Omega_{T}\right)}=O\left(\varepsilon^{-\frac{1}{2}}\right) .
$$

Estimates (5.11) give the same convergence rates than (4.4) and (4.5) for equations (DD-EI). They are optimal due to a similar example given in Section 4. Now we describe the boundary layers of the above limit in one space dimension. To this end, we use all the notations introduced in Sections 3-4. Let $\left(n^{\varepsilon}, \phi^{\varepsilon}\right)$ and $(n, \phi)$ be solutions of the problems (5.1)-(5.5) and (5.6)-(5.10) respectively. Let $(n(t, 0)+u(t, y), \phi(t, 0)+\varphi(t, y))$ and $(n(t, 1)+v(t, z), \phi(t, 1)+\psi(t, z))$ be approximations of $\left(n^{\varepsilon}, \phi^{\varepsilon}\right)$ in a neighborhood of $\Gamma_{0}=\{x=0\}$ and $\Gamma_{1}=\{x=1\}$ respectively, where $y=x / \varepsilon$ and $z=(1-x) / \varepsilon$. Then, similar to the analysis given in Section 3 , 
we obtain the boundary layer equations for $(u, \varphi)$ and $(v, \psi)$ :

$$
\begin{aligned}
\partial_{y y} \varphi(t, y) & =f_{e}\left(a_{e}(t)+\mu_{e} \varphi(t, y)\right)-f_{i}\left(\mu_{i} \phi(t, 0)-\mu_{i} \varphi(t, y)\right) \text { in } B_{T}, \\
\varphi(t, 0) & =\varphi_{b l}(t), \lim _{y \rightarrow+\infty} \varphi(t, y)=0 \text { in }[0, T], \\
u(t, y) & =f_{i}\left(a_{i}(t)-\mu_{i} \varphi(t, y)\right)-f_{i}\left(a_{i}(t)\right) \text { in } B_{T}, \\
\partial_{z z} \psi(t, z) & =f_{e}\left(b_{e}(t)+\mu_{e} \psi(t, z)\right)-f_{i}\left(\mu_{i} \phi(t, 1)-\mu_{i} \psi(t, z)\right) \text { in } B_{T}, \\
\psi(t, 1) & =\psi_{b l}(t), \lim _{z \rightarrow+\infty} \psi(t, z)=0 \text { in }[0, T], \\
v(t, z) & =f_{i}\left(b_{i}(t)-\mu_{i} \psi(t, z)\right)-f_{i}\left(b_{i}(t)\right) \text { in } B_{T},
\end{aligned}
$$

where $\phi_{b l}, \psi_{b l}, a_{i}$ and $b_{i}$, are defined in (3.5), (3.14), (3.6) and (3.15) respectively, which are continuous functions on $[0, T]$. Since

$$
f_{i}\left(a_{i}(t)\right)=f_{e}\left(\mu_{e} \phi(t, 0)\right)=n(t, 0), \quad f_{i}\left(b_{i}(t)\right)=f_{e}\left(\mu_{e} \phi(t, 1)\right)=n(t, 1),
$$

by a similar argument to the proof of Theorem 3.1, we obtain the existence and uniqueness of the boundary layers with exponential decay to 0.

Theorem 5.2. Under the assumptions and the notations of Theorem 5.1, the boundary layer equations (5.12)(5.14) (respectively (5.15)-(5.17)) admit a unique solution $(u, \varphi)$ (respectively $(v, \psi)$ ), which is continuous in $t$, of class $C^{3}$ and monotone in $y$ (respectively $z$ ), such that for $(t, y) \in[0, T] \times(0,+\infty)$

$$
|u(t, y)|,|\varphi(t, y)|,\left|\partial_{y} u(t, y)\right|,\left|\partial_{y} \varphi(t, y)\right| \leq C_{\varphi}\left|\varphi_{b l}(t)\right| \exp \left(-\delta_{\varphi} y\right)
$$

and for $(t, z) \in[0, T] \times(0,+\infty)$

$$
|v(t, z)|,|\psi(t, z)|,\left|\partial_{z} v(t, z)\right|,\left|\partial_{z} \psi(t, z)\right| \leq C_{\psi}\left|\psi_{b l}(t)\right| \exp \left(-\delta_{\psi} z\right) .
$$

\section{Appendix. Proof of Lemma 4.1}

To show (4.4), we use the Poisson equation

$$
\begin{aligned}
& -\varepsilon^{2} \partial_{x x} \phi^{\varepsilon}=n_{i}^{\varepsilon}-n_{e}^{\varepsilon}, \\
& \phi^{\varepsilon}=\phi_{D} \text { on } \Sigma_{0} \cup \Sigma_{1},
\end{aligned}
$$

where

$$
\Sigma_{0}=(0, T) \times\{x=0\}, \quad \Sigma_{1}=(0, T) \times\{x=1\} .
$$

Since the sequence $\left(n_{\alpha}^{\varepsilon}\right)_{\varepsilon>0}$ is bounded in $L^{\infty}\left(\Omega_{T}\right)$ for $\alpha=i, e$, we deduce easily that $\left(\left.\varepsilon^{2} \partial_{x} \phi^{\varepsilon}\right|_{\Gamma_{D}}\right)_{\varepsilon>0}$ is bounded in $L^{\infty}(0, T)$. 
In the sequel, we denote by $C_{i}\left(i \in \mathbb{N}^{*}\right)$ various positive constants independent of $\varepsilon$. Let $\Gamma_{D}=\Gamma_{0} \cup \Gamma_{1}$. Using again the Poisson equation, the definition of $R^{\varepsilon}$ and (4.1)-(4.3), we have

$$
\begin{aligned}
& \left\|R^{\varepsilon}\right\|_{L^{2}\left(\Omega_{T}\right)}^{2}=\int_{\Omega_{T}}\left(R^{\varepsilon}\right)^{2} \mathrm{~d} x \mathrm{~d} t \\
& =\int_{\Omega_{T}}\left(n_{i}^{\varepsilon}-n_{e}^{\varepsilon}+u_{e}^{\varepsilon}+v_{e}^{\varepsilon}-u_{i}^{\varepsilon}-v_{i}^{\varepsilon}\right) R^{\varepsilon} \mathrm{d} x \mathrm{~d} t \\
& =-\varepsilon^{2} \int_{\Omega_{T}} \partial_{x x} \phi^{\varepsilon} R^{\varepsilon} \mathrm{d} x \mathrm{~d} t+\int_{\Omega_{T}}\left(u_{e}^{\varepsilon}+v_{e}^{\varepsilon}-u_{i}^{\varepsilon}-v_{i}^{\varepsilon}\right) R^{\varepsilon} \mathrm{d} x \mathrm{~d} t \\
& \leq \varepsilon^{2} \int_{\Omega_{T}} \partial_{x} \phi^{\varepsilon} \partial_{x} R^{\varepsilon} \mathrm{d} x \mathrm{~d} t-\left.\varepsilon^{2} \int_{0}^{T} \partial_{x} \phi^{\varepsilon}(t) R^{\varepsilon}(t)\right|_{\Gamma_{D}} \mathrm{~d} t \\
& +\left\|u_{e}^{\varepsilon}+v_{e}^{\varepsilon}-u_{i}^{\varepsilon}-v_{i}^{\varepsilon}\right\|_{L^{2}\left(\Omega_{T}\right)}\left\|R^{\varepsilon}\right\|_{L^{2}\left(\Omega_{T}\right)} \\
& \leq \varepsilon^{2} \int_{\Omega_{T}} \partial_{x} \phi^{\varepsilon} \partial_{x}\left(n_{i}^{\varepsilon}-n_{e}^{\varepsilon}\right) \mathrm{d} x \mathrm{~d} t+\varepsilon^{2} \int_{\Omega_{T}} \partial_{x} \phi^{\varepsilon} \partial_{x}\left(u_{e}^{\varepsilon}+v_{e}^{\varepsilon}-u_{i}^{\varepsilon}-v_{i}^{\varepsilon}\right) \mathrm{d} x \mathrm{~d} t \\
& +4 C_{1} \varepsilon^{\frac{1}{2}}\left\|R^{\varepsilon}\right\|_{L^{2}\left(\Omega_{T}\right)}+C_{2} \exp (-\delta / \varepsilon),
\end{aligned}
$$

where $\delta=\min \left(\delta_{\varphi}, \delta_{\psi}\right)$. Hence, by Young's inequality and the following basic inequality for small $\varepsilon$ :

$$
\exp (-\delta / \varepsilon) \leq C_{2} \varepsilon
$$

we obtain

$$
\left\|R^{\varepsilon}\right\|_{L^{2}\left(\Omega_{T}\right)}^{2} \leq 2 \varepsilon^{2} \int_{\Omega_{T}} \partial_{x} \phi^{\varepsilon} \partial_{x}\left(n_{i}^{\varepsilon}-n_{e}^{\varepsilon}\right) \mathrm{d} x \mathrm{~d} t+2 \varepsilon^{2} \int_{\Omega_{T}} \partial_{x} \phi^{\varepsilon} \partial_{x}\left(u_{e}^{\varepsilon}+v_{e}^{\varepsilon}-u_{i}^{\varepsilon}-v_{i}^{\varepsilon}\right) \mathrm{d} x \mathrm{~d} t+C_{3} \varepsilon .
$$

The first term on the right hand side of $(*)$ can be estimated as in [14]. Indeed, from (2.2) we may write

$$
n_{i}^{\varepsilon}=f_{i}\left(F_{i}^{\varepsilon}-\mu_{i} \phi^{\varepsilon}\right), n_{e}^{\varepsilon}=f_{e}\left(F_{e}^{\varepsilon}+\mu_{e} \phi^{\varepsilon}\right) .
$$

Therefore, from the lower and upper bounds for $\left(n_{\alpha}^{\varepsilon}\right)_{\varepsilon>0}$ and the $L^{2}\left(0, T ; H^{1}(\Omega)\right)$ bounds for $\left(F_{\alpha}^{\varepsilon}\right)_{\varepsilon>0}$, we have

$$
\begin{aligned}
\varepsilon^{2} \int_{\Omega_{T}} \partial_{x} \phi^{\varepsilon} \partial_{x}\left(n_{i}^{\varepsilon}-n_{e}^{\varepsilon}\right) \mathrm{d} x \mathrm{~d} t= & \varepsilon^{2} \int_{\Omega_{T}}\left[f_{i}^{\prime}\left(F_{i}^{\varepsilon}-\mu_{i} \phi^{\varepsilon}\right) \partial_{x} \phi^{\varepsilon} \partial_{x} F_{i}^{\varepsilon}-f_{e}^{\prime}\left(F_{e}^{\varepsilon}+\mu_{e} \phi^{\varepsilon}\right) \partial_{x} \phi^{\varepsilon} \partial_{x} F_{e}^{\varepsilon}\right] \mathrm{d} x \mathrm{~d} t \\
& -\varepsilon^{2} \int_{\Omega_{T}}\left[\mu_{i} f_{i}^{\prime}\left(F_{i}^{\varepsilon}-\mu_{i} \phi^{\varepsilon}\right)+\mu_{e} f_{e}^{\prime}\left(F_{e}^{\varepsilon}+\mu_{e} \phi^{\varepsilon}\right)\right]\left(\partial_{x} \phi^{\varepsilon}\right)^{2} \mathrm{~d} x \mathrm{~d} t \\
\leq & \frac{\varepsilon^{2}}{2} \int_{\Omega_{T}}\left[f_{i}^{\prime}\left(h_{i}\left(n_{i}^{\varepsilon}\right)\right)\left(\partial_{x} F_{i}^{\varepsilon}\right)^{2} / \mu_{i}+f_{e}^{\prime}\left(h_{e}\left(n_{e}^{\varepsilon}\right)\right)\left(\partial_{x} F_{e}^{\varepsilon}\right)^{2} / \mu_{e}\right] \mathrm{d} x \mathrm{~d} t \\
& -\frac{\varepsilon^{2}}{2} \int_{\Omega_{T}}\left[\mu_{i} f_{i}^{\prime}\left(h_{i}\left(n_{i}^{\varepsilon}\right)\right)+\mu_{e} f_{e}^{\prime}\left(h_{e}\left(n_{e}^{\varepsilon}\right)\right)\right]\left(\partial_{x} \phi^{\varepsilon}\right)^{2} \mathrm{~d} x \mathrm{~d} t \\
\leq & C_{4} \varepsilon^{2}-C_{4} \varepsilon^{2}\left\|\partial_{x} \phi^{\varepsilon}\right\|_{L^{2}\left(\Omega_{T}\right)}^{2} .
\end{aligned}
$$

Here we have used the relations:

$$
f_{\alpha}^{\prime}\left(h_{\alpha}\left(n_{\alpha}^{\varepsilon}\right)\right)=\frac{1}{h_{\alpha}^{\prime}\left(n_{\alpha}^{\varepsilon}\right)}=\frac{n_{\alpha}^{\varepsilon}}{p_{\alpha}^{\prime}\left(n_{\alpha}^{\varepsilon}\right)} \geq C_{4} / \mu_{\alpha}, \quad \alpha=i, e .
$$


For the second term on the right hand side of $(*)$, using again Young's inequality and Theorem 3.1, we have

$$
\begin{aligned}
\varepsilon^{2}\left|\partial_{x} \phi^{\varepsilon} \partial_{x}\left(u_{e}^{\varepsilon}+v_{e}^{\varepsilon}-u_{i}^{\varepsilon}-v_{i}^{\varepsilon}\right)\right| & \leq \frac{C_{4} \varepsilon^{2}}{2}\left(\partial_{x} \phi^{\varepsilon}\right)^{2}+C_{5} \varepsilon^{2}\left[\partial_{x}\left(u_{e}^{\varepsilon}+v_{e}^{\varepsilon}-u_{i}^{\varepsilon}-v_{i}^{\varepsilon}\right)\right]^{2} \\
& \leq \frac{C_{4} \varepsilon^{2}}{2}\left(\partial_{x} \phi^{\varepsilon}\right)^{2}+2 C_{5} \varepsilon^{2}\left[\partial_{x}\left(u_{e}^{\varepsilon}-u_{i}^{\varepsilon}\right)\right]^{2}+2 C_{5} \varepsilon^{2}\left[\partial_{x}\left(v_{e}^{\varepsilon}-v_{i}^{\varepsilon}\right)\right]^{2} \\
& =\frac{C_{4} \varepsilon^{2}}{2}\left(\partial_{x} \phi^{\varepsilon}\right)^{2}+2 C_{5}\left[\partial_{y}\left(u_{e}-u_{i}\right)\right]^{2}+2 C_{5}\left[\partial_{z}\left(v_{e}-v_{i}\right)\right]^{2} \\
& \leq \frac{C_{4} \varepsilon^{2}}{2}\left(\partial_{x} \phi^{\varepsilon}\right)^{2}+2 C_{5} C_{0}^{2}\left[\exp \left(-2 \delta_{\varphi} y\right)+\exp \left(-2 \delta_{\psi} z\right)\right],
\end{aligned}
$$

which yields

$$
\varepsilon^{2} \int_{\Omega_{T}} \partial_{x} \phi^{\varepsilon} \partial_{x}\left(u_{e}^{\varepsilon}+v_{e}^{\varepsilon}-u_{i}^{\varepsilon}-v_{i}^{\varepsilon}\right) \mathrm{d} x \mathrm{~d} t \leq \frac{C_{4} \varepsilon^{2}}{2}\left\|\partial_{x} \phi^{\varepsilon}\right\|_{L^{2}\left(\Omega_{T}\right)}^{2}+C_{6} \varepsilon
$$

Hence

$$
\left\|R^{\varepsilon}\right\|_{L^{2}\left(\Omega_{T}\right)}^{2}+\frac{C_{4} \varepsilon^{2}}{2}\left\|\partial_{x} \phi^{\varepsilon}\right\|_{L^{2}\left(\Omega_{T}\right)}^{2} \leq C_{7} \varepsilon
$$

and thus (4.4) is proved.

Acknowledgements. An improved version of this article was finished during the visit of the author in IMS of the Chinese University of Hong Kong. He thanks Prof. Z.P. Xin for his hospitality and valuable discussions.

\section{REFERENCES}

[1] J.P. Aubin, Un théorème de compacité. C. R. Acad. Sci. Paris 256 (1963) 5042-5044.

[2] Y. Brenier, Convergence of the Vlasov-Poisson system to the incompressible Euler equations. Comm. Partial Differential Equations 25 (2000) 737-754.

[3] H. Brézis, F. Golse, R. Sentis, Analyse asymptotique de l'équation de Poisson couplée à la relation de Boltzmann. Quasineutralité des plasmas. C. R. Acad. Sci. Paris 321 (1995) 953-959.

[4] S. Cordier, P. Degond, P. Markowich, C. Schmeiser, Traveling wave analysis and jump relations for Euler-Poisson model in the quasineutral limit. Asymptot. Anal. 11 (1995) 209-224.

[5] S. Cordier, E. Grenier, Quasineutral limit of an Euler-Poisson system arising from plasma physics. Comm. Partial Differential Equations 25 (2000) 1099-1113.

[6] P.C. Fife, Semilinear elliptic boundary value problems with small parameters. Arch. Rational Mech. Anal. 52 (1973) $205-232$.

[7] H. Gajewski, On the uniqueness of solutions to the drift-diffusion model of semiconductor devices. Math. Models Methods Appl. Sci. 4 (1994) 121-133.

[8] I. Gasser, The initial time layer problem and the quasi-neutral limit in a nonlinear drift diffusion model for semiconductors. Nonlinear Differential Equations Appl. (to appear).

[9] I. Gasser, D. Levermore, P. Markowich, C. Schmeiser, The initial time layer problem and the quasi-neutral limit in the drift-diffusion model (submitted).

[10] A. Jüngel, A nonlinear drift-diffusion system with electric convection arising in semiconductor and electrophoretic modeling. Math. Nachr. 185 (1997) 85-110.

[11] A. Jüngel, Y.J. Peng, A hierarchy of hydrodynamic models for plasmas. Zero-relaxation-time limits. Comm. Partial Differential Equations 24 (1999) 1007-1033.

[12] A. Jüngel, Y.J. Peng, A hierarchy of hydrodynamic models for plasmas. Zero-electron-mass limits in the drift-diffusion equations. Ann. Inst. H. Poincaré, Anal. Non Linéaire 17 (2000) 83-118.

[13] A. Jüngel, Y.J. Peng, Zero-relaxation-time limits in hydrodynamic models for plasmas revisited. Z. Angew. Math. Phys. 51 (2000) 385-396.

[14] A. Jüngel, Y.J. Peng, A hierarchy of hydrodynamic models for plasmas. Quasi-neutral limits in the drift-diffusion equations. Asymptot. Anal. (to appear). 
[15] J.L. Lions, Quelques méthodes de résolution des problèmes aux limites non linéaires. Dunod-Gauthier-Villard, Paris (1969).

[16] P.A. Markowich, A singular perturbation analysis of the fundamental semiconductor device equations. SIAM J. Appl. Math. 44 (1984) 896-928.

[17] P.A. Markowich, C. Ringhofer, C. Schmeiser, An asymptotic analysis of one-dimensional models for semiconductor devices. IMA J. Appl. Math. 37 (1986) 1-24.

[18] Y.J. Peng, Convergence of the fractional step Lax-Friedrichs scheme and Godunov scheme for a nonlinear Euler-Poisson system. Nonlinear Anal. TMA 42 (2000) 1033-1054.

[19] P. Raviart, On singular perturbation problems for the nonlinear Poisson equation or: A mathematical approach to electrostatic sheaths and plasma erosion, Lect. Notes of the Summer school in Ile d'Oléron, France (1997) 452-539.

[20] L. Tartar, Compensated compactness and applications to partial differential equations. In: Nonlinear analysis and mechanics: Heriot-Watt Symp. Vol. 4 and Res. Notes Math. 3 (1979) 136-212.

[21] A. Visintin, Strong convergence results related to strict convexity. Comm. Partial Differential Equations 9 (1984) $439-466$.

To access this journal online: www.edpsciences.org 